\title{
Simulation of Glancing Shock Wave and Boundary Layer Interaction
}

Ching-Mao Hung, Ames Research Center, Moffett Field, California

\section{N/SN}

National Aeronautics and

Space Administration 
$\cdots$ 


\title{
Simulation of Glancing Shock Wave and Boundary Layer Interaction
}

\section{CHING-MAO HUNG ${ }^{1}$}

\author{
NASA Ames Research Center, Moffett Field, CA
}

\begin{abstract}
After months of fishing with a net and carefully studying, a marine professor concluded that 1) all fishes have gills, and 2) no fish is smaller than two inches.
\end{abstract}

\section{Abstract}

Shock waves generated by sharp fins, glancing across a laminar boundary layer growing over a flat plate, are simulated numerically. Several basic issues concerning the resultant three-dimensional flow separation are studied. Using the same number of grid points, different grid spacings are employed to investigate the effects of grid resolution on the origin of the line of separation. Various shock strengths ( generated by different fin angles) are used to study the so-called separated and unseparated boundary layer and to establish the existence or absence of the secondary separation. The usual interpretations of the flow field from previous studies and new interpretations arising from the present simulation are discussed.

\section{INTRODUCTION}

In the past, fluid dynamics has been divided into two branches, theoretical and experimental. Continuing advances in numerical methods and in computer capabilities have at some point qualified computation as a separate branch of fluid dynamics. Using the computer as a tool, computational fluid dynamics (CFD) is able to supplement the other two branches and to carry out its own research, developinent, and further advancement in fluid dynamics as a field of physical science.

A shock wave generated by one body, glancing across a boundary layer on another body, is a problem well suited to CFD capabilities. This is one of the most common and important three-dimensional (3-D) inviscid/viscous interaction problems. The problem is so complicated that a purely theoretical approach is almost impossible. In early studies, investigators have relied mainly on experimental observations of surface properties, such as static pressure and oil flow, to deduce the basic interaction features of the flow. With the advent of the supercomputer, we are now on the threshold of definitive explorations, finding the details of the flow-field structure and the underlying physical processes.

There are various kinds of glancing shock wave problems. Instead of dealing problens involving complicated geometries, the present paper will focus on the problem of a supersonic flow over a sharp fin mounted on a flat plate, shown in Fig. 1. Recently this simple geometry has attracted a substantial amount of interest and has been studied extensively, (for example, Refs. 1 - 5). However, there are still many flow-field features that need better explanations.

The primary purpose of this paper is to study several basic issues regarding the resultant 3-D flow separation. The separation of the boundary layer on the flat plate, ( the sidewall), under the influence of the glancing shock wave will be specially emphasized. To avoid the uncertainty associated with turbulent modeling in our study, the flow is assumed to be laminar. The compressible Navier-Stokes equations will be solved. Cases of sharp fins

\footnotetext{
${ }^{1}$ Research Scientist, Computational Fluid Dynamics Branch.
} 
with various wedge angles will be investigated numerically. The results are first compared with the experimental and computational results of Degrez $[3]$ for $M_{\infty}=2.25, R e=10^{5}$ and a wedge angle of $6^{\circ}$. Results for wedge angles of $2^{\circ}$ and $12^{\circ}$ will also be discussed. Using the same number of grid points, different grid spacings are employed to study the effects of grid resolution on the origin of the line of separation. Various shock strengths generated by different fin angles are used to study the so-called separated and unseparated boundary layer and the existence or absence of a secondary separation. The usual interpretations from previous studies and new interpretations arising from the present simulation will be discussed.

It should be mentioned that this type of flow field, when turbulent, is observed to be unsteady (see discussion in Ref. 6). However, in the present study, the flow is assumed laminar and steady. The applicability of the present discussion to the turbulent cases is based upon the assumption that the random turbulence fluctuations are suppressed and the turbulent flow is steady in a "mean" sense.

\section{NUMERICAL PROCEDURE}

The governing equations of the present analysis are the time-dependent, compressible Navier-Stokes equations incorporating the concept of the thin-layer approximation in all three directions (Baldwin and Lomax [7]). The flow is assumed laminar and the wall is adiabatic. A numerical procedure developed by Hung and Kordulla [8] without time-splitting is used. The basic numerical scheme is MacCormack's [9] explicit-implicit predictor-corrector algorithm. The solution is carried out until it converges to a steady state. Details of the numerical technique and boundary conditions are discussed in Ref. 8 .

Figure 2 shows a typical mesh system of $57 \times 45 \times 27$ points for a sharp fin on a flat plate. The apex of the fin (at $\mathrm{x}=0.0$ ) is placed at a distance $L=9 \mathrm{~cm}$ from the flat plate leading edge and this distance $L$ is used in the characteristic length in the present study. Here $(x, y, z)$ and $(I, J, K)$ are used in the conventional sense of streamwise, crossflow, and vertical directions. The domain of computation lies in the intervals $-1.0 \leq x \leq 3.5$, $0.0 \leq y \leq 4.5$, and $0.0 \leq z \leq 1.5$. Using the same number of grid points, three different grid spacing are employed. The coarse grid is uniform in the streamwise direction and geometrically stretched from the fin and plate (in the J-and $\mathrm{K}$-directions). The medium grid has additional geometric stretching in the streamwise direction from the apex of the fin. The fine grid has finer spacing, ( compared to the medium grid ), near the fin in the Jdirection and near the apex of the fin in the streamwise direction. To avoid over-stretching in the outer region, several zones with different stretching factors are used in the I- and J-directions. Smooth transition in grid spacing is ensured from one zone to another. The grid spacing parameters are listed in Table 1.

\section{RESULTS AND DISCUSSIONS}

The flow to be simulated has free-stream Mach number $M_{\infty}=2.25$, Reynolds number $R e=10^{5}$, and free-stream temperature $T_{\infty}=263^{\circ} \mathrm{R}$. The results are at first compared with the experimental and computational results of Degrez for a wedge angle $\theta=6^{\circ}$. Figure 3 shows a comparison of surface pressures at $y=5 \mathrm{~cm}$. All results are in good agreement, except that the computational result of Degrez shows a "dip" after the separation. The present results and the experimental data do not show the appearance of the dip in surface pressure. The fine grid result (not shown in Fig. 3) is very close to the medium grid result. This indicates that our grid refinement does not affect the prediction of surface pressure. 


\section{Origin of the Line of Separation}

Reference 4 contains an extensive study of the flow-field structure of this geometry for turbulent boundary layer. It is well accepted that the primary separation is a consequence of the high pressure, recovered from the shock system, which induces flow from the fin surface and forces the boundary layer off the sidewall. The question is, where is the origin of the line of primary separation? Figure 4 shows particle traces of the result based on a two-equation model as described in Ref. 4 for the first mesh points above the sidewall ( $\mathrm{K}=2$ ). ( The figure is provided by Horstman [10]. ) This particle trace is constructed by a time integration of velocity components restricted to the plane of $\mathrm{K}=2$. Since the plane of $\mathrm{K}=2$ is very close to the flat plate, (normally it would have a resolution smaller than the size of an oil particle), the particle integrations are treated as surface particle traces and are considered equivalently as a simulation of oil flow in the experiment and as a simulation of skin-friction lines in the theoretical approach. The "oil flow" in Fig. 4 indicates that the line of separation originates somewhere in the plate away from the apex of the fin and that this feature is an open-type separation. (An open-type separation contains only regular points, while a closed-type separation originates from a saddle point, as discussed in Refs. 11 and 12.)

Figures $5 \mathrm{a}-5 \mathrm{c}$ show surface particle traces for the sequence of three grid refinements. For the coarse grid, the separation is an open type. As the grid spacing near the leading edge is refined, the starting point of the open-type separation moves and eventually the separation becomes a closed type. This clearly demonstrates that the grid resolution can affect the "calculated" topology. The coarse grid simply cannot resolve the vortex structure, while the fine grid can. As the wedge angle increases to $12^{\circ}$ the vortex structure is large enough that the medium grid (not shown here) is able to reveal a closed-type separation.

While the existence or not of an open-type separation is still an unanswered question, we believe that some of the numerically observed open-type separations (for instance, Fig. 4) result from insufficient grid resolution. Similarly, every experiment also has resolution problems, such as the size of oil droplets. Some of the experimentally observed open-type separations may be the result of low resolution of the device and facility.

We would like to point out that the concept of a closed 3-D separated region being inaccessible (see Ref. 11) is valid only in the limit of particles moving near/on body surface. The upstream flow particles above the surface are able to access the separated region behind the line of separation through the spiral nature of the separation. Indeed, there is no 3-D separation which is totally closed by a separation surface, as a closed bubble; there must be some fluid flowing in and some fluid flowing out. All 3-D separation surfaces are a kind of vortex sheet.

Hereafter the fine-grid result will be used for discussion, except for cases specially mentioned.

\section{Secondary Separation}

The second question is the existence of a secondary separation. Experiments (for instance Ref. 13) very often show the appearance of another oil-accumulation line behind the line of primary separation. This has been interpreted as indicating the existence of a secondary separation, ( see Fig. 6). The plots of velocity at the first mesh point above the flat plate, $K=2$, (Fig. 7a) also show that, in addition to the outermost primary separation line, (not clearly visible on the figure), there is a second "line" of clustering or coalescence of velocity vectors. However, based on the result of surface particle traces (Fig. 7b) this is not a line of separation - it is merely a demarcation between regions 
of high and low surface skin friction. One might imagine that, in a transient stage of an experiment, comparatively more "oil" can be driven in by the high skin friction from the right and less oil carried out to the left near the region of strong variation in skin friction. (For convenience of discussion, here left or right refers to the orientation of one facing the streamwise direction.) Hence the surface may show a temporary accumulation of oil around this second line. Even as the wedge angle increases up to $12^{\circ}$ (Fig. 8), our calculations still show no evidence of the existence of secondary separation. Indeed for the $12^{\circ}$ case, behind the obvious primary separation line, there is a region that the particle traces show strongly convergent from one side and slowly divergent away from the other side. (These traces eventually converge to the primary separation line.) Hence a high clustering of particle traces in that region occurs. From the plot of velocity vectors in the plane $\mathrm{K}=2$ (Fig. 9a), one can see that this region is associated with the drastic change of the surface skin friction. 'The flow features near the wall are strongly affected by the surface pressure. Their relations and connections can be seen from Fig. 9b. A strong surface pressure gradient induces a high skin friction and a high velocity near the sidewall. This results in a divergence of particle traces and leads to an appearance of the so-called attachment line (Fig. 9b). After the strong pressure gradient, there is a region of drastic change of skin friction and hence a resulting appearance of clustering of velocity vectors near the wall. The strong pressure gradient appears mainly on the right of the inviscid shock location (Fig. 9b) and the clustering of velocity on the left (Fig. 9a). Note that the appearance of the clustering of particle traces (Fig. 8) does not coincide with the clustering of the velocity vectors near the wall. Instead, showing as a band with the clustering of the velocity vectors on its right, it is close to the pressure plateau region (see below). It is this pressure plateau region that causes the particle traces to run almost parallel to each other before they finally converge to the primary separation line. We would suspect that variations in surface-flow-visualization techniques would also result in different locations of temporary clustering of surface-streak lines.

Note that Degrez's calculation showed a noticeable 'dip' of pressure for the $6^{\circ}$ case (see Fig. 4). When it is strong enough, this drop in pressure can significantly retard the primary separated flow (passing beneath the shock system in the opposite y-direction to the main flow) and lead to a secondary separation (see Fig. 12 of Ref. 3). In the present calculation, the pressure shows a plateau region and there is no secondary separation for either the $6^{\circ}$ or $12^{\circ}$ case. (The $12^{\circ}$ case has a large plateau region with a little dip of pressure.) As discussed above a drastic change of velocity leads to a substantial change of skin friction which might also result in a temporary accumulation of oil flow on the surface. Therefore, it is possible that an accumulation of oil flow on the surface in an experiment is not necessarily a line of separation contrary to the usually inference. This argument has also arisen in previous experiments and calculations for other geometries $[14,15]$.

A note of caution should be given here. We don't know whether the appearance of a secondary oil-accumulation in an experiment is caused by a secondary separation or not. One possible alternative is suggested here. Furthermore, there might be other mechanisms in an experiment, especially for the turbulent case, that could lead to an oil accumulation on the surface. Further detailed and careful studies are needed to answer these questions.

\section{Absence of Separation}

Whether the boundary layer on the plate is separated or not is usually determined by comparing the turning angle of the limiting streamline with the glancing shock angle in the interaction region (Fig. 10). As shown in Figs. 5 and 8, there is clearly a line, with clustering of particle traces, that originates from a saddle point with turning angle greater than the angle of the glancing shock and the flows are separated. As the wedge angle decreases, one would expect that the turning angle of the skin friction line will decrease 
and eventually become smaller than the angle of the glancing shock. The flow then will be classified as attached What is the change of the flowfield topology from attached to separated flow? This question is addressed in the following section.

In the present paper a case with $2^{\circ}$ wedge angle was calculated with the fine grid distribution. Fig. 11a shows traces of particles for which the origins are almost the same as those of the $6^{\circ}$ case (Fig. 5 ). In contrast to Figs. 5 and 8 , there is no obvious line of convergence of particle traces and the turning of skin-friction lines is smaller than the glancing shock angle; this would conventionally be interpreted as an attached case. However, a close examination of the particle traces near the apex (Fig. 11b) shows that actually the flow is separated. Even though it is very small, the separation also is a closed type, and the structure is topologically the same as that for the previous $6^{\circ}$ and $12^{\circ}$ cases. Actually, all three cases are topologically the same as the structure of a blunt-fin flow field.

One may imagine that, under certain conditions, this type of flow may not separate. However, the conventional method of interpretation using the turning angle of the boundary layer compared to the glancing shock angle as a criterion for separation is not uniformly valid, as demonstrated above. To date, the simplest and most general definition of a 3-D separation line, in the opinion of the author is that of Legendre [16]; "a line of separation .. . has no local property. Its only characteristic is to pass through a saddle point" (There may be doubt as to whether or not there is a local property as pointed out in Refs. 17 and 18.)

Another point should be mentioned here. Figs. 12 and 21 of Ref. 5 showed that, at a low wedge angle, based on the oil-flow picture the boundary layer on the sidewall was separated from a saddle point, as a closed type of separation, on the line of symmetry near the fin apex, and then gradually became attached away from the fin. (This is not an open-type separation as claimed in Ref. 5.) The question arises as to where and how the separation ends. In the opinion of the present author the simplest explanation is that this is case in which the definition of separation based on turning angle and oil-accumulation in experimental observation fails. Because of boundary layer growth on the fin, the shock wave is stronger and hence the pressure rise is higher near the leading edge of the fin than at a position downstream. The difference in pressure rise changes the turning angle and degree of oil accumulation. Based on the concept of continuity, limiting streamlines would not join together except at a singular point. A line of separation, once it originates from a saddle point, will either continue going downstream to infinity or terminate at a singular point. In the other words, once it is separated, the flow can not gradually be reattached without a singular point, according to topological imperatives.

\section{Separation on Fin Surface}

As sketched in Fig. 12 of Ref. 5, even at low wedge angle there is a separation on the fin surface, as the high pressure flow near the fin surface tries to flow across onto the sidewall. The question arises as to where and how the separation starts. For a boundary layer to separate, an adverse pressure gradient is necessary. Plots of particle traces restricted to the plane of $\mathrm{J}=2$ and surface pressure are shown in Figs. 12a and $12 \mathrm{~b}$ for the $12^{\circ}$ case. In Fig. $12 \mathrm{a}$, in addition to the vortex spiral nature of the primary separation, we can see that the separation starts from a saddle point (a closed type). Correspondingly, there is a low pressure region and an adverse pressure gradient that triggers the separation (Fig. 12b). By overlaying Fig. 12a with Fig. 12b, one can see that the separation line is downstream of ( or on the righthand side of), not coincident with, the pressure minimum along each limiting streamline. The existence of a low pressure region can be attributed to the gradual decrease of total pressure in the incoming boundary layer and hence a decease of the pressure rise after the shock (see Fig. 12b). The small increase in pressure near the 
plate results from flow stagnation. Topologically, there is even a 'hoseshoe vortex' (Fig. 12a) as in the blunt-fin solution, but it is too weak to induce a significant pressure gradient.

\section{CONCLUSION AND REMARKS}

A laminar supersonic flow over a sharp fin mounted on a flat plate has been numerically simulated. Separation of the boundary layer on the flat plate was investigated for various grid refinements and fin wedge angles. Several basic issues concerning 3-D steady separation have been discussed.

The results of the solution have demonstrated that grid resolution can affect the "calculated" topology. For the coarse grid, the separation is an open type. As the grid spacing near the leading edge is refined, the starting point of the open-type separation moves and eventually the separation becomes a closed type. In the opinion of the author, some of the numerically observed open-type separations (for example, Fig. 4) results from insufficient grid resolution. Similarly, experiments have resolution problems, and some of the experimentally observed open-type separations may well be due to low resolution of the device and facility.

Based on the computation of surface particle traces, no secondary separation is found in the present study. A secondary oil-accumulation line has been conjectured to be a demarcation between regions of high and low surface skin friction.

In a calculation with a $2^{\circ}$ wedge angle, there is no obvious line of convergent particle traces and the turning angles of skin-friction lines are smaller than the glancing shock angle. This is conventionally interpreted as an attached flow. However, a close examination of the particle traces near the apex has shown that actually the flow is separated, and the structure is the same topologically as that for the blunt-fin flow field.

\section{REFERENCES}

[1] Hung, C. M. and MacCormack, R. W., "Numerical solution of three-dimensional shock-wave and turbulent boundary-layer interaction," AIAA J., Vol. 16, No. 12 Dec. (1978), pp. 1090-1096.

[2] Horstman, C. C. and Hung, C. M., "Computation of 3-D turbulent separated flow at supersonic speed," AIAA Paper No. 79-0002, (1979).

[3] Degrez, G.,"Computation of a three-dimensional skewed shock wave laminar boundary layer interaction," AIAA Paper No. 85-1565, (1985).

[4] Knight, D. D., Horstman, C. C., Shapey, B., and Bogdonoff, S., "The flowfield structure of the 3-D shock wave-boundary layer interaction generated by a $20^{\circ}$ sharp fin at Mach 3," AIAA Paper No. 86-0343, (1986).

[5] Fomison, N. R. and Stollery, J. L.,"The effects of sweep and bluntness on a glancing shock wave turbulent boundary layer interaction," AGARD CP 428, paper 8, (1987).

[6] Dolling, D.S.,"Unsteadiness of supersonic and hypersonic shock-induced turbulent boundary layer separation," AGARD-FDP/VKI Special Course on "Three-Dimensional Supersonic and Hypersonic Flows Including Separation", May 8-12, 1989.

[7] Baldwin, B. S. and Lomax, H., "Thin-layer approximation and algebraic model for separated turbulent flows," AIAA paper No. 78-257, Jan. (1978).

[8] Hung, C. M. and Kordulla, W., "A time-split finite-volume algorithm for threedimensional flowfield simulation," AIA A J., Vol. 22, No. 11, (1984), pp. 1564-1572. 
[9] MacCormack, R.W. "A numerical method for solving the equations of viscous flow," AIAA J., Vol. 20, No. 9, (1982), pp. 1275-1281.

[10] Horstman, C. C., private communication.

[11] Wang, K.C.,"Boundary layer separation in three dimensions," Reviews in Viscous Flow, Proc of the Lockheed - Georgia Company Viscous Flow Symposium, June 1976, pp. 341-414.

[12] Tobak, M. and Peake, D. J., "Topology of three-dimensional separation flows," NASA TM-81294, April 1981.

[13] Aso, S., Hayashi, M., and Tan, A. Z., "The structure of aerodynamic heating in threedimensional shock wave / turbulent boundary layer induced by sharp and blunt fins," AIAA paper no. 89-1854, June (1989).

[14] Hung, C.M., "Computation of three-dimensional shock wave and boundary layer interactions," NASA TM-86780, Aug. 1985.

[15] Hung, C.M., "Computation of separation ahead of blunt fin in supersonic turbulent flow," NASA TM-89416, Dec. 1986.

[16] Legendre, R., "Regular or catastrophic evolution of steady flows depending on parameters," Rech. Aerosp. Vol. 1982-4, pp. 41-49, (1982).

[17] Zhang, H. X., "The separation criteria and flow behavior for three dimensional steady separated flow," translated from ACTA Aerodynamica Sinica, March (1985), pp. 112 .

[18] Wu, J.Z., Gu, J.W., and Wu, J.M.,"Steady three-dimensional fluid particle separation from arbitrary smooth surface and formation of free vortex layers," AIAA Paper No. 87-2348,July (1987).

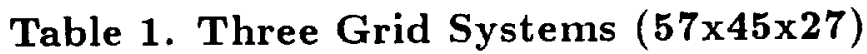

\begin{tabular}{|c|c|c|c|}
\hline & coarse & medium & fine \\
\hline$\triangle x$ min & 0.10 & 0.02 & .01 \\
\hline$\triangle y$ min & 0.0025 & 0.0025 & 0.0015 \\
\hline$\triangle z$ min & 0.001 & 0.001 & 0.001 \\
\hline plate leading edge & $\mathrm{I}=4$ & $\mathrm{I}=3$ & $\mathrm{I}=3$ \\
\hline fin leading edge & $\mathrm{I}=14$ & $\mathrm{I}=22$ & $\mathrm{I}=22$ \\
\hline
\end{tabular}




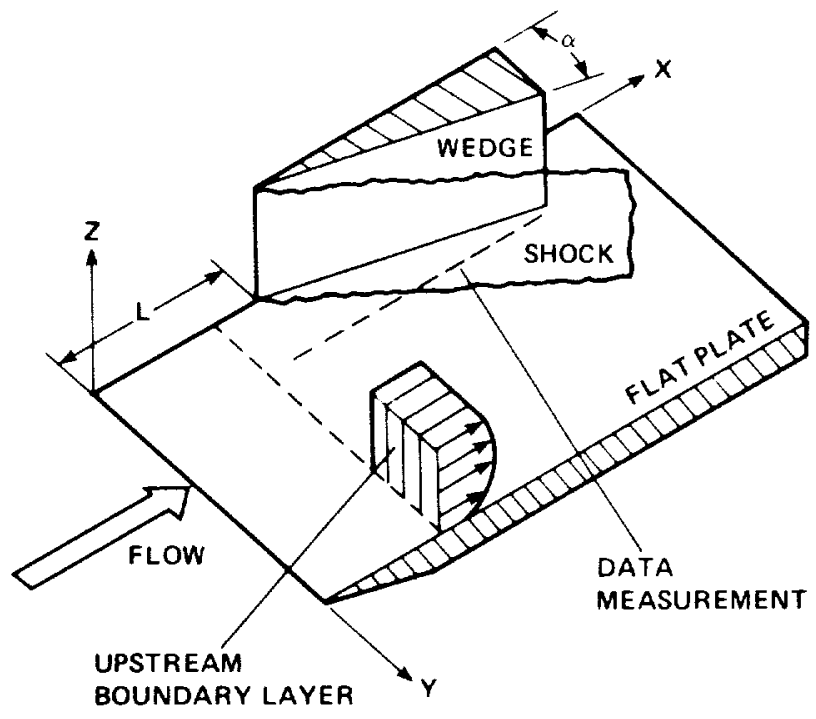

Fig. 1 Glancing shock wave over a boundary layer.

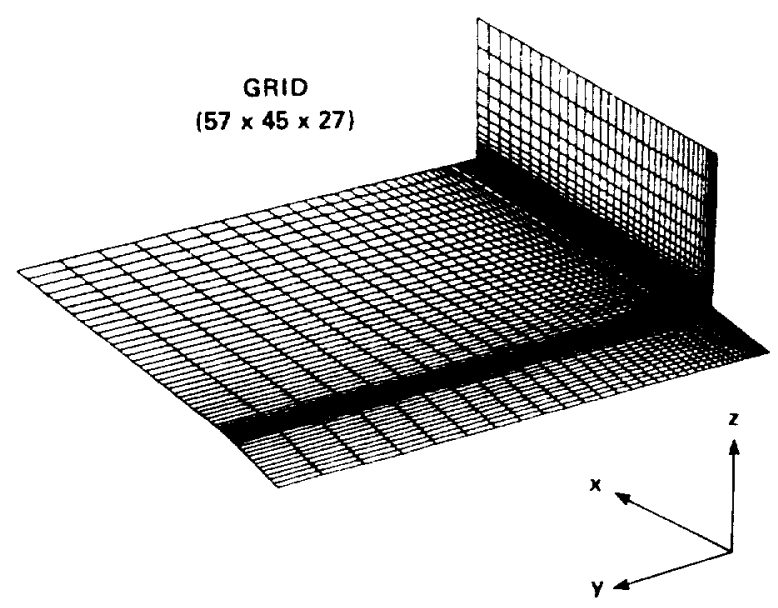

Fig. 2 Mesh system in fin and flat plate.

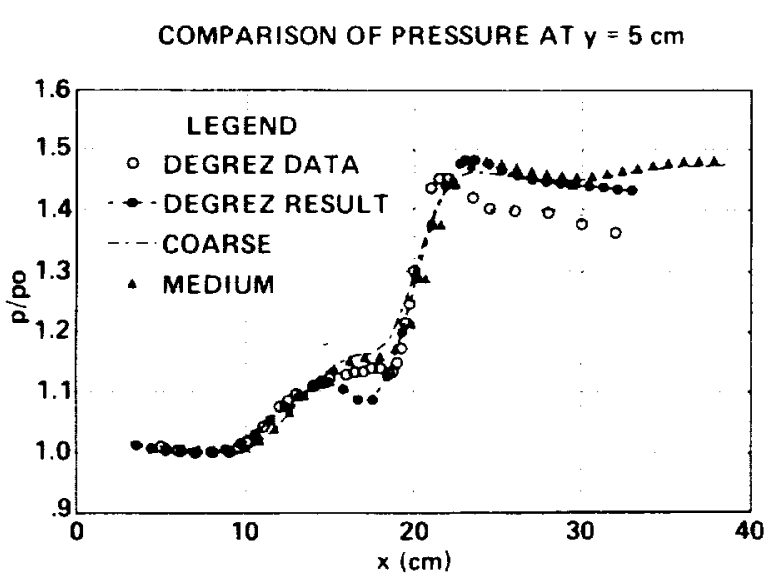

Fig. 3 Comparison of surface pressure at $\mathrm{y}=5 \mathrm{~cm}$.

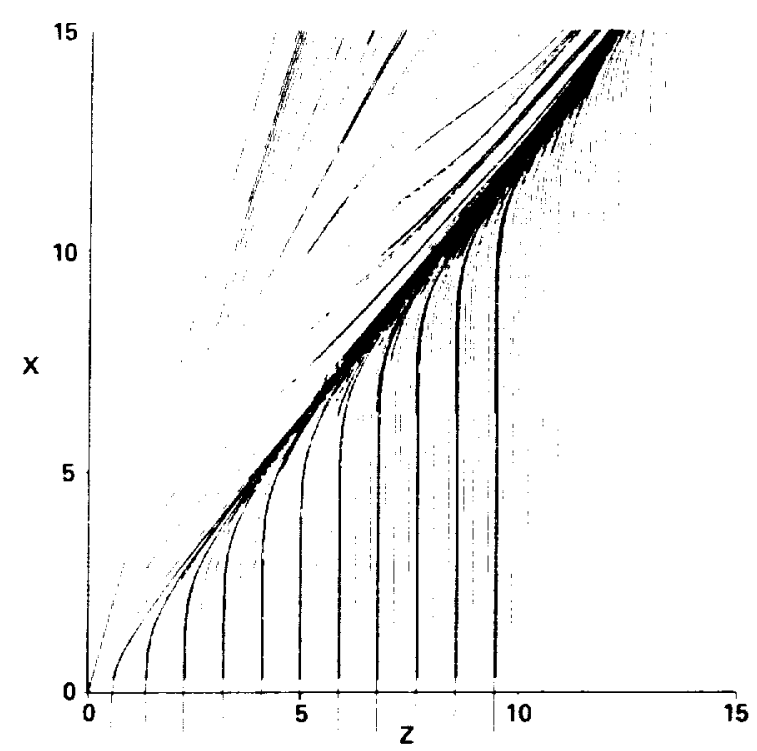

Fig. 4 Surface particle traces from Horstman (Ref. 4)
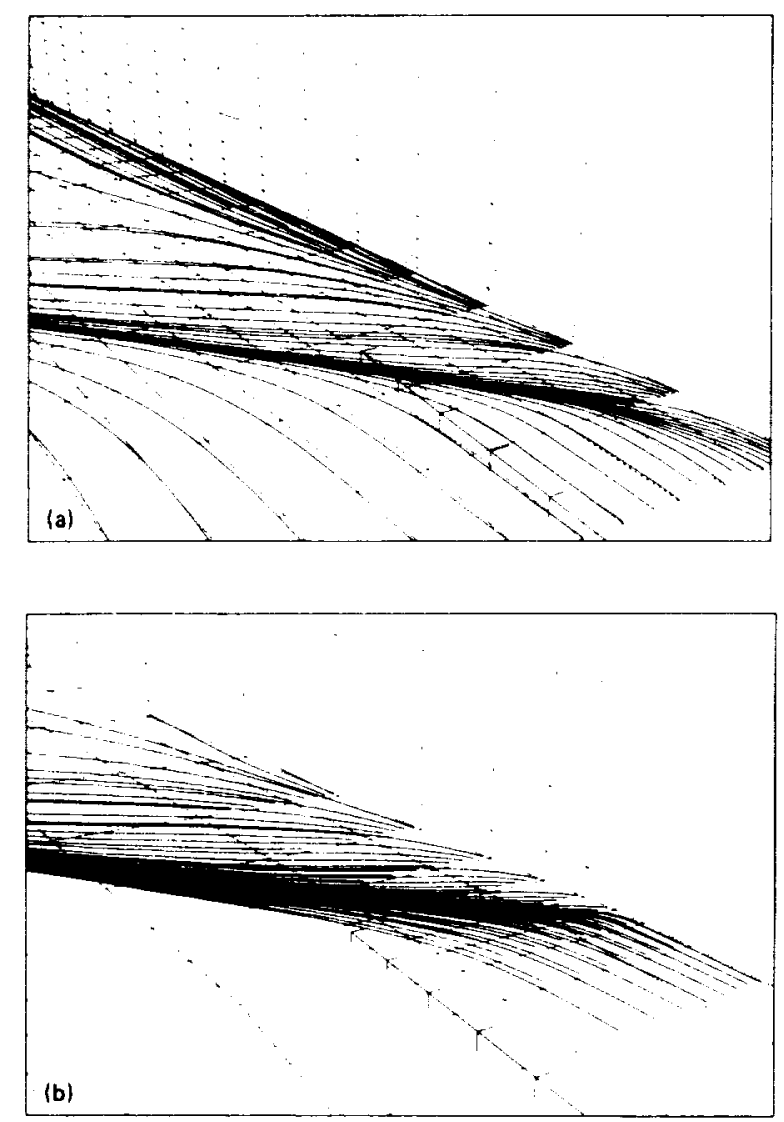

Fig. 5 Surface particle traces $(\mathrm{K}=2)$ for $\alpha=6^{\circ}$ : (a) Coarse grid; (b) medium grid. 


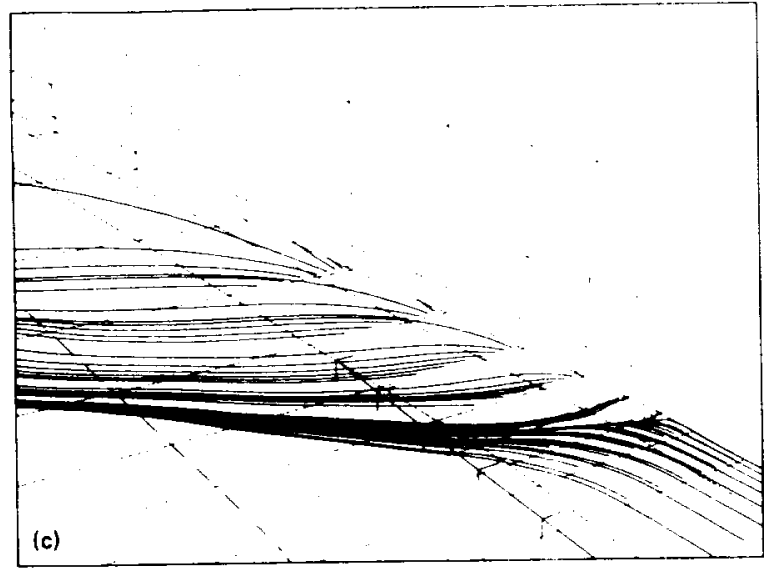

Fig. 5 Concluded. (c) Fine grid.

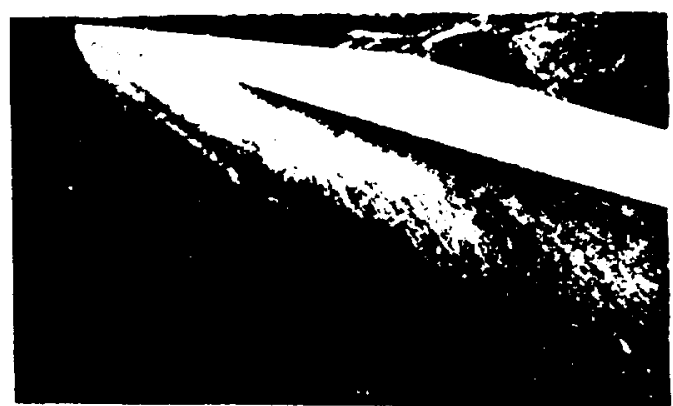

(a) OIL FLOW

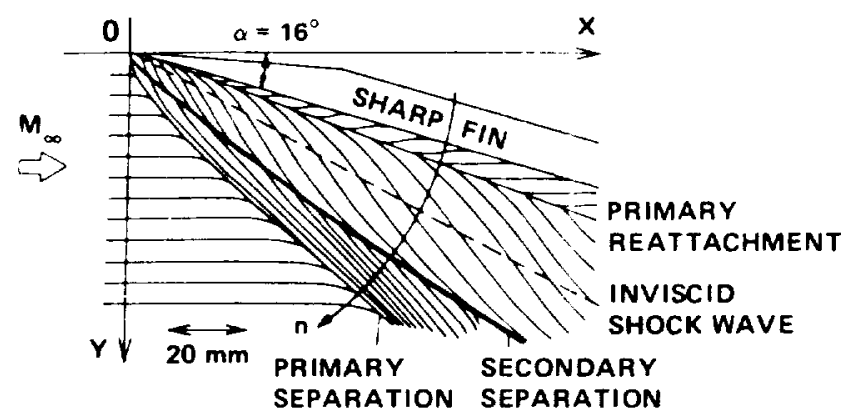

(b) SKETCH OF OIL STREAK LINES

Fig. 6 Surface oil flow by Aso, etc. (Ref. 13).
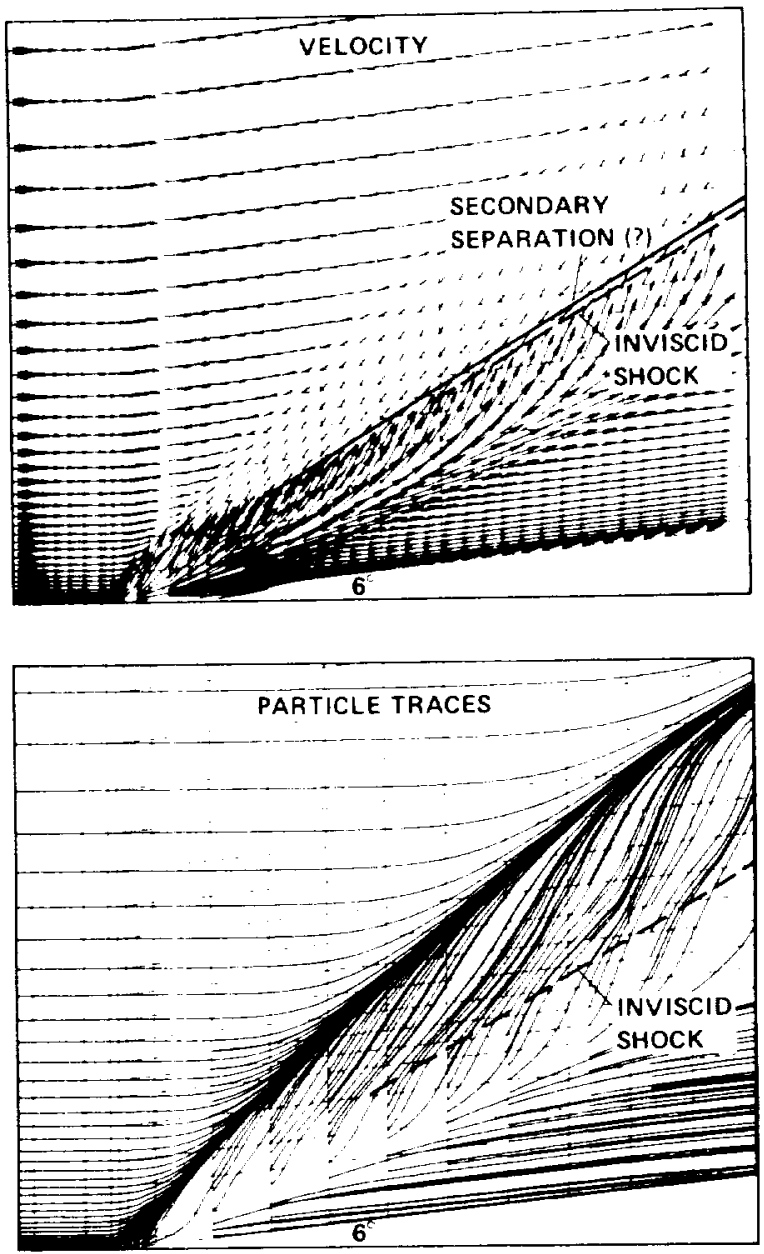

Fig. 7 Velocity vectors and particle traces for $\mathrm{K}=2\left(\alpha=6^{\circ}\right)$ : (a) Velocity vectors; (b) particle traces.

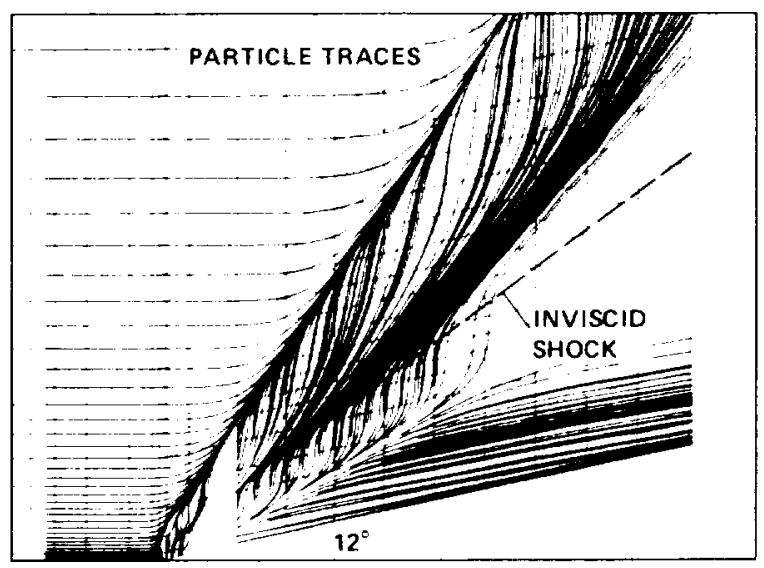

Fig. 8 Surface particle traces $(K=2)$ for $\alpha=12^{\circ}$. 

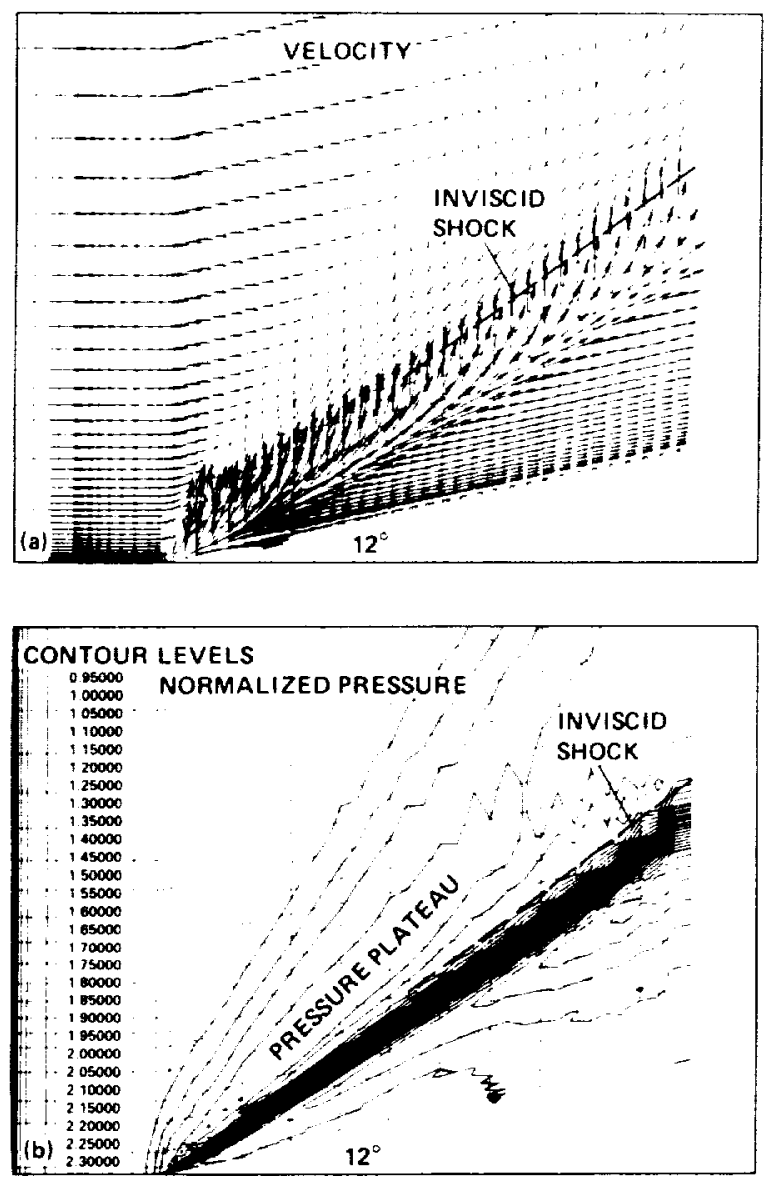

Fig. 9 Velocity vectors and pressure contours for $\mathrm{K}=2\left(\alpha=6^{\circ}\right)$ : (a) Velocity vectors; (b) pressure contours.
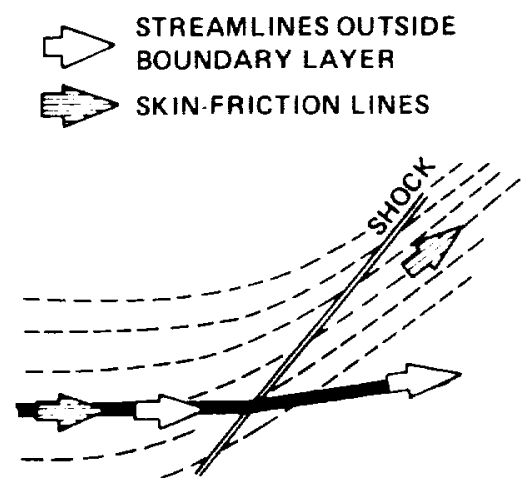

(a) ATTACHED FLOW

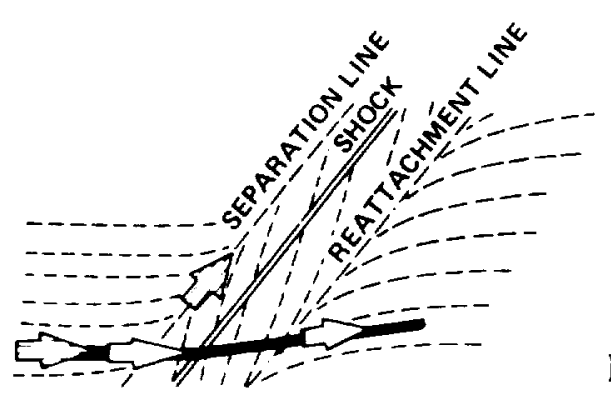

(b) SEPARATED FLOW

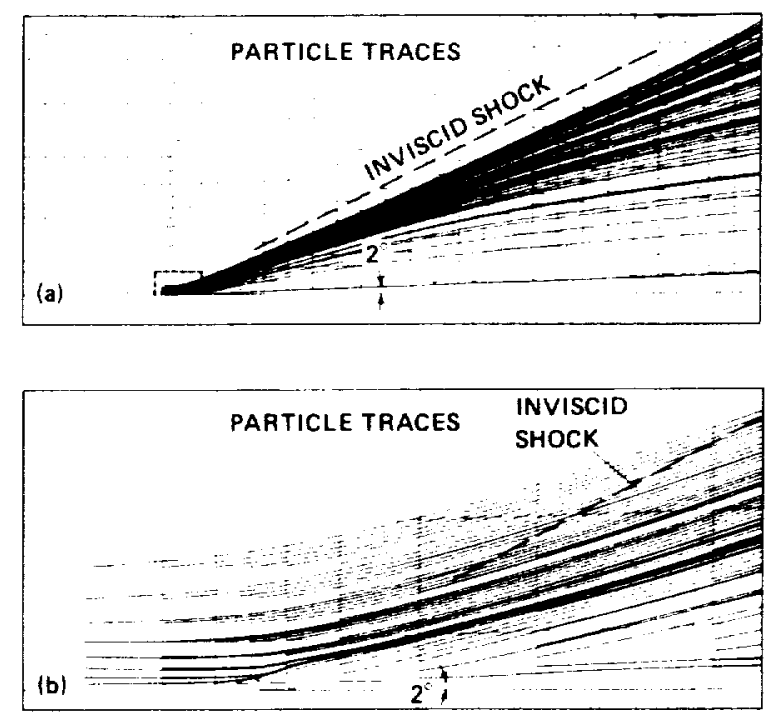

Fig. 11 Surface particle traces $(K=2)$ for $\alpha=2^{\circ}$ : (a) General feature; (b) near apex
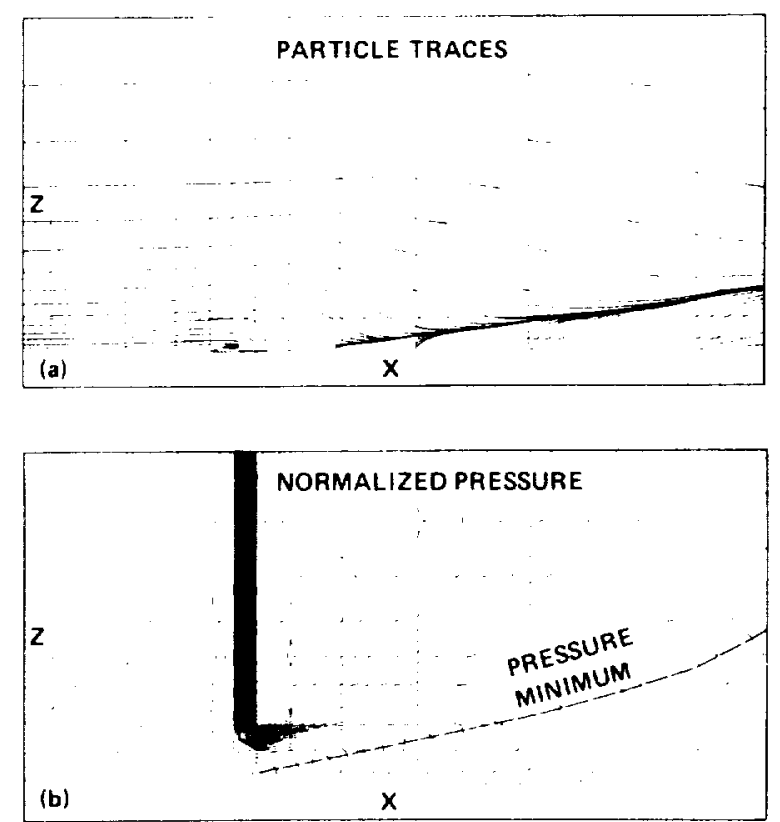

Fig. 12 Particle traces and pressure contours on the fin surface $(J=2)$ for $\alpha=2^{\circ}$ : (a) Particle traces; (b) pressure contours
Fig. 10 Sketches of skin-friction line patternsbeneath glancing shock 


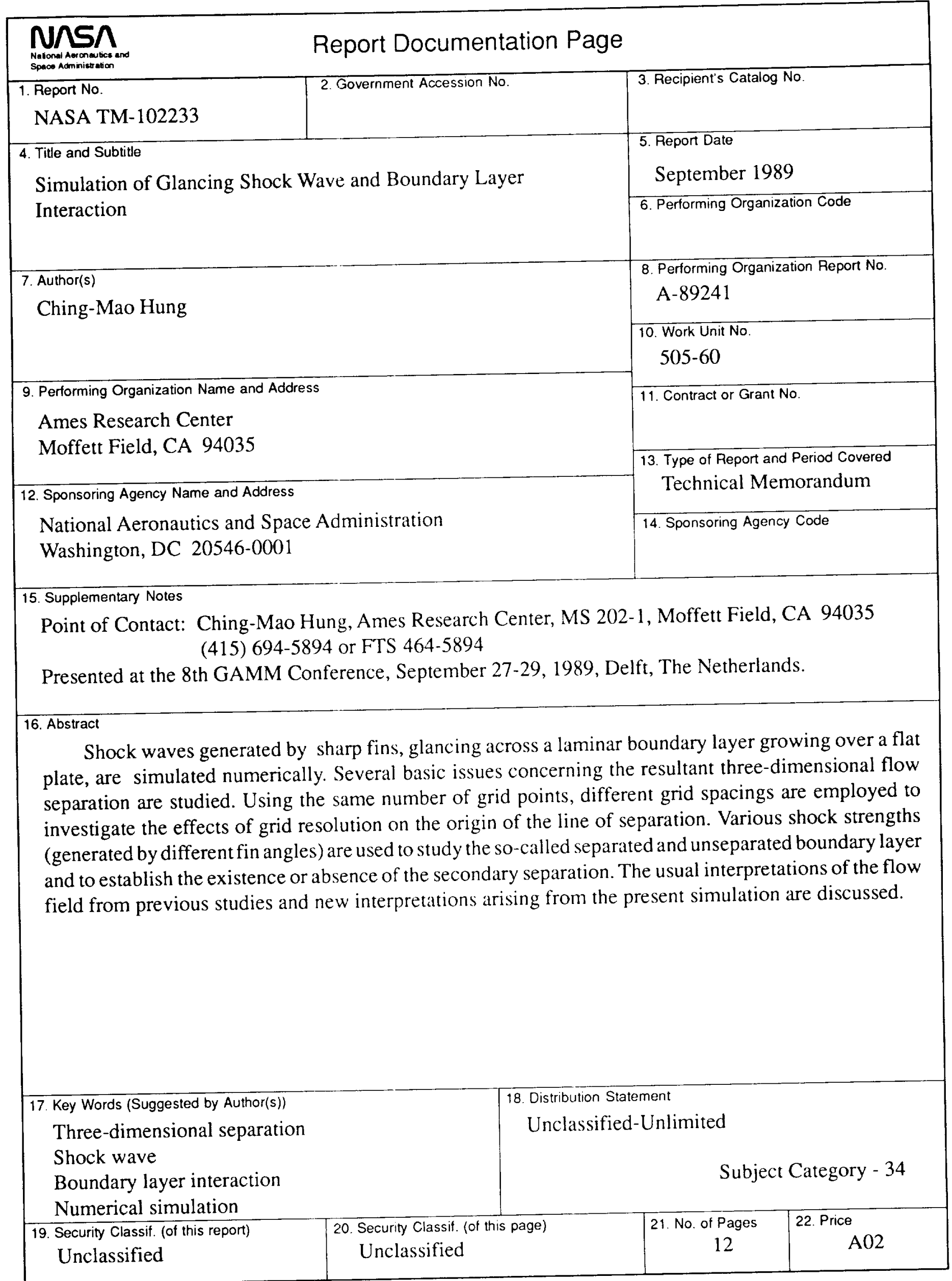


